\title{
Ocular Manifestations in Granulomatosis with Polyangiitis Patients from Saudi Arabia
}

\author{
Abdurhman Saud Al Arfaj ${ }^{*}$ (1), Najma Khalil1,2 \\ ${ }^{1}$ Department of Medicine, Rheumatology Division, College of Medicine, King Saud University, Riyadh, KSA \\ ${ }^{2}$ College of Medicine Research Center, College of Medicine, King Saud University, Riyadh, KSA \\ Email: *asarfaj@ksu.edu.sa
}

How to cite this paper: Al Arfaj, A.S. and Khalil, N. (2021) Ocular Manifestations in Granulomatosis with Polyangiitis Patients from Saudi Arabia. Open Journal of Rheumatology and Autoimmune Diseases, 11, 9-18.

https://doi.org/10.4236/ojra.2021.111002

Received: December 22, 2020

Accepted: February 1, 2021

Published: February 4, 2021

Copyright $\odot 2021$ by author(s) and Scientific Research Publishing Inc. This work is licensed under the Creative Commons Attribution International License (CC BY 4.0).

http://creativecommons.org/licenses/by/4.0/

\begin{abstract}
Eye and/or orbit involvement occurs in Granulomatosis with polyangiitis (GPA) patients frequently. The aim of our study was to describe the clinical manifestations, therapy and outcome of ocular involvement in our GPA patients. A retrospective study was conducted including patients with GPA who followed up in Rheumatology clinics during 1990-2016 at King Khalid University Hospital, Riyadh. Information relating to demographics, ocular manifestations, laboratory findings, therapy and outcome of GPA patients were noted. Ocular involvement was detected in $9(39.1 \%)$ of the 23 GPA cases identified. The mean age of ocular GPA patients was 51.8 (range 27 - 62) years, the mean age at onset of disease was 39.6 (range $11-57$ ) years and the mean duration of disease was 9.0 (range 2 - 19) years. Concomitant ear, nose, throat and sino-nasal manifestations occurred with ocular symptoms in $77.8 \%$ GPA patients. The most frequent manifestations were, eye pain (66.7\%), scleritis/episcleritis (55.6\%), eye redness and itching (55.6\% each). Antineutrophil cytoplasmic antibodies (ANCA) were positive in $88.9 \%$ patients, $55.6 \%$ had c-ANCA and $33.3 \%$ had p-ANCA. Infections were observed in $22.2 \%$ of patients, which included pneumonia in one patient and esophageal candidiasis and bacterial meningitis in another. All patients received oral prednisolone, $44.4 \%$ received intravenous cyclophosphamide, $22.2 \%$ refractory cases received rituximab doses and the disease outcome was good. Comparison of ocular GPA with non-ocular GPA patients showed that $77.8 \%$ of ocular GPA patients had concomitant sino-nasal symptoms compared to $42.9 \%$ in non-ocular GPA patients and $22.2 \%$ of the ocular GPA patients had renal involvement compared to $64.3 \%$ in non-ocular GPA patients $(\mathrm{p}=$ 0.049). We found that the frequency of ocular manifestations in our GPA patients was similar to reports elsewhere, and the most frequent symptom was eye pain and scleritis/episcleritis.
\end{abstract}




\section{Keywords}

Granulomatosis with Polyangiitis, Ocular Manifestations, Orbital Involvement, Lacrimal Gland Involvement

\section{Introduction}

Granulomatosis with polyangiitis (GPA) is an autoimmune granulomatous inflammatory disease that affects multiple organs and is characterized by small vessel necrotizing vasculitis. It predominantly affects upper and lower respiratory tracts and kidneys [1] [2]. Ophthalmic involvement of eye and/or orbit occurs in up to $60 \%$ of GPA patients and is the initial manifestation in $8 \%-16 \%$ [3] [4] [5] [6]. The eye is considered an immune-privileged site, with a high predisposition to develop anti-inflammatory and immunosuppressive mechanisms to prevent the consequences of inflammation [7]. The most common clinical findings are proptosis, scleritis, episcleritis, retinal, and optic nerve vasculitis, nasolacrimal duct obstruction, uveitis, and dacryocystitis [3] [8]. Permanent blindness may occur in $8 \%$ of the patients [1] [4]. GPA is the most common rheumatic disease to affect the orbit [9]. Orbital lesions are reported in 5\% - 31\% GPA patients, which can be bilateral in $14 \%$ - 58\% while the lacrimal gland may be involved initially [1] [4] [10]-[15].

Our aim was to study the ocular manifestations in GPA patients from our region, its disease course, therapy and the outcome.

\section{Materials and Methods}

The medical charts of all GPA patients diagnosed and followed up in Rheumatology clinics at King Khalid University Hospital, King Saud University, Riyadh, during the period 1990-2016 were reviewed retrospectively. GPA was diagnosed as per Chapel Hill Consensus Conference (CHCC) definition [16]. Study approval was obtained from Institutional Review Board of College of Medicine, King Saud University, Riyadh. GPA patients who underwent ophthalmic evaluation for ocular symptoms and were diagnosed to have ocular manifestations of GPA were included in the present study. We recorded the demographic data including, patient's age, age at disease onset, gender, duration of disease, interval between onset of symptoms and diagnosis and duration of follow up. Clinical, hematological and immunological data were also retrieved. Eye and orbital/lacrimal gland computed tomography (CT) and biopsy findings were noted. Antineutrophil cytoplasmic antibodies (ANCA) were detected by indirect immunofluorescence (for detecting c-ANCA and p-ANCA) and enzyme-linked immunosorbent assay (ELISA) for detecting proteinase 3 (PR3) and/or myeloperoxidase (MPO) ANCA). Treatment given to the patients, disease course and its outcome were also recorded.

Statistical Analysis: Statistical analysis of the data was performed using IBM 
SPSS statistics for Windows Version 19.0. (Armonk, NY: IBM Corp) and presented as percentages and means. We compared ocular GPA with non-ocular GPA groups using chi-square and t-tests and a p-value $<0.05$ was considered statistically significant.

\section{Results}

Twenty-three cases of GPA were included in this study. Eye was involved in nine (39.1\%) patients (ocular-GPA) and the remaining 14 patients did not show eye symptoms (non-ocular GPA). Of the nine ocular-GPA patients, seven (77.8\%) were males and two (22.2\%) were females (male: female; 3.5:1). Mean present age $( \pm \mathrm{SD}$ ) was $51.8 \pm 12.6$ (range 27 - 62) years, mean age at onset of disease was $39.6 \pm 17.2$ (range $11-57$ ) years, mean interval between onset of symptoms and diagnosis was $7.3 \pm 11.2$ (range 0 - 30) months, mean duration of disease was 9.0 \pm 5.3 (range 2 - 19) years and mean duration of follow up was $5.5 \pm 3.96$ (range 0.8 - 10.8) years.

Constitutional symptoms occurred in three (33.3\%) patients including fatigue in one (11.1\%), fever in three (33.3\%), anorexia in one (11.1\%) and weight loss in two (22.2\%) patients. Arthralgia was the presenting symptom in four (44.4\%) patients and none had lymphadenopathy. Other systems involved were; ENT in seven $(77.7 \%)$, skin in two (22.2\%), pulmonary in six (66.7\%), renal in two (22.2\%), neurological in one (11.1\%) and gastrointestinal in one $(11.1 \%)$ patient. Skin involvement was in the form of palpable purpuric lesions. Various eye manifestations are summarized in Table 1. Eye pain (66.7\%) and scleritis/episcleritis Table 1. Eye symptoms in 9 ocular-GPA patients.

\begin{tabular}{cc}
\hline Eye symptoms & No. (\%) \\
Eye pain & $6(66.7)$ \\
Eyelid swelling & $3(33.3)$ \\
Eye redness & $5(55.6)$ \\
Eye itching & $5(55.6)$ \\
Eye discharge & $1(11.1)$ \\
Conjunctivitis & $4(44.4)$ \\
Blurred vision & $1(11.1)$ \\
Cataract & $1(11.1)$ \\
Orbital and lacrimal gland swelling & $1(11.1)$ \\
Scleritis & $1(11.1)$ \\
Nodular scleritis & $2(22.2)$ \\
Episcleritis & $2(22.2)$ \\
Uveitis & $1(11.1)$ \\
Bilateral myopic degeneration & $1(11.1)$ \\
Peripheral corneal infiltrates & $2(22.2)$ \\
Retinal detachment & $1(11.1)$ \\
\hline
\end{tabular}


(55.6\%) constituted the most frequent eye symptoms followed by conjunctivitis (44.4\%). One patient had recurrent episcleritis with bilateral myopic degeneration and one patient had corneal infiltrates with retinal detachment and vasculitis.

Laboratory findings in nine ocular-GPA patients are summarized in Table 2. ANCA was positive in eight (88.9\%) patients and negative in one patient. Among the eight ANCA positive patients, c-ANCA was positive in five (55.6\%) and p-ANCA was positive in three (33.3\%) patients. PR3 ANCA was positive in all four patients tested and MPO was negative.

Eye CT showed granuloma. Upper eyelid and lacrimal gland biopsy showed non-necrotizing granulomatous vasculitis. One patient had lacrimal gland drainage and another patient had surgery performed for nodular scleritis.

Patients were admitted to hospital for intravenous cyclophosphamide (IV CYC) infusion, rituximab administration, disease flares and infections. Two patients $(22.2 \%)$ had infections which included pneumonia in one and esophageal candidiasis and bacterial meningitis in the other patient. They were on corticosteroids and IV CYC treatment. One patient was admitted to intensive care unit for evaluation of pulmonary symptoms flare up, bilateral pleural effusion and pulmonary lesion. All patients were given oral prednisolone (PSL), four (44.4\%) patients received IV CYC and two (22.2\%) patients received rituximab doses (Table 3). There were no deaths and all patients were in remission on treatment at the time of follow up.

The results of comparison of ocular GPA with non-ocular GPA group of patients are presented in Table 4. There were more males compared to females $(7: 2)$ in ocular GPA group while equal number of males and females were affected in non-ocular GPA (7:7). The proportions of ear nose throat (ENT) and

Table 2. Laboratory parameters in 9 Ocular GPA patients.

\begin{tabular}{cc}
\hline Lab Parameters & No. (\%) \\
Leukocytosis & $2(22.2)$ \\
Anemia & $3(33.3)$ \\
Thrombocytopenia & $0(0.0)$ \\
Elevated ESR & $6(66.7)$ \\
Elevated serum creatinine & $2(22.2)$ \\
Abnormal creatinine clearance & $2(22.2)$ \\
ANCA Positive & $8(88.9)$ \\
ANCA Negative & $1(11.1)$ \\
c ANCA Positive & $5(55.6)$ \\
$\mathrm{p}$ ANCA Positive & $3(33.3)$ \\
PR3 Positive $(\mathrm{n}=4)($ not done $=4)$ & $4(100.0)$ \\
PR3 Negative $(\mathrm{n}=4)($ not done $=4)$ & $0(0.0)$ \\
MPO Positive $(\mathrm{n}=4)($ not done $=4)$ & $0(0.0)$ \\
MPO Negative $(\mathrm{n}=4)($ not done $=4)$ & $4(100.0)$ \\
\hline
\end{tabular}


Table 3. Therapy in 9 ocular-GPA patients.

\begin{tabular}{cc}
\hline Therapy & No. (\%) \\
Prednisolone & $9(100.0)$ \\
Methyl Prednisolone & $3(33.3)$ \\
Oral Cyclophosphamide & $4(44.4)$ \\
Intravenous Cyclophosphamide & $4(44.4)$ \\
Azathioprine & $4(44.4)$ \\
Methotrexate & $4(44.4)$ \\
Infliximab & $1(11.1)$ \\
Rituximab & $2(22.2)$ \\
\hline
\end{tabular}

Table 4. Comparison of ocular GPA and non-ocular GPA patients.

\begin{tabular}{cccc}
\hline Characteristic & Ocular GPA $(\mathbf{n}=9)$ & Non-Ocular GPA $(\mathbf{n}=14)$ & p value \\
\hline Age at disease onset (years) & & & \\
Mean \pm SD & $39.6 \pm 17.2$ & $37.3 \pm 17.6$ & 0.893 \\
Range & $11-57$ & $11-63$ & \\
M:F ratio & $3.5: 1$ & $1: 1$ & 0.311 \\
ENT involvement & $7(77.8)$ & $8(57.1)$ & 0.111 \\
Sini nasal involvement & $7(77.8)$ & $6(42.9)$ & 0.493 \\
Cutaneous involvement & $2(22.2)$ & $5(35.7)$ & 0.907 \\
Pulmonary involvement & $6(66.7)$ & $9(64.3)$ & $0.049^{*}$ \\
Renal involvement & $2(22.2)$ & $9(64.3)$ & 0.742 \\
Neurological involvement & $1(11.1)$ & $1(7.1)$ & 0.493 \\
Elevated Serum creatinine & $2(22.2)$ & $5(35.7)$ & 0.742 \\
ANCA positive & $8(88.9)$ & $13(92.9)$ & 0.231 \\
c-ANCA positive & $5(55.6)$ & $12(85.7)$ & 0.231 \\
p-ANCA positive & $3(33.3)$ & $1(7.1)$ &
\end{tabular}

${ }^{*} \mathrm{p}$ value significant.

sino-nasal involvement were higher in ocular GPA (77.8\% each) compared to non-ocular GPA $(57.1 \%, 42.9 \%)$ patients; however, they were not statistically significant. Similarly, c-ANCA were more prevalent in non-ocular GPA patients (85.7\%) compared to ocular GPA patients (55.6\%), though not significant statistically $(\mathrm{p}>0.05)$. There was significant association of renal involvement with non-ocular GPA patients (64.3\%) compared with ocular GPA (22.2\%) patients $(\mathrm{p}=0.049)$.

\section{Discussion}

Ocular involvement has been reported to occur in 14\% - 61\% GPA patients [1] [6] [17] [18] [19]. The frequency of $39.1 \%$ in our patients falls within this range. It is known to occur in both limited and severe forms of GPA and can be the ini- 
tial symptom or occur in as high as $87 \%$ GPA patients at some time during the disease [1] [20] [21]. However, GPA with limited involvement of the orbit and/or the eye is seen rarely [22]. The Spanish registry of systemic vasculitis study reported that $24 \%$ of GPA patients had ocular involvement at diagnosis [23]. Ocular GPA often presents as stromal keratitis, blurred vision, and scleritis/episcleritis [7] [24] [25]. In our study scleritis/episcleritis was the most frequent ocular manifestation, which occurred in $55.6 \%$ patients compared to $10 \%-87 \%$ in previous reports [1] [5] [7] [25] [26] [27]. Scleritis in GPA patients can be nodular, diffuse or necrotizing [27]. Our patients presented with nodular scleritis. Scleritis is reported to lead to vision loss and blindness if not adequately treated [27] [28]. Vision loss or total blindness has been reported in 4.3\% - 37\% GPA patients [7] [29]. Blurred vision was noted in $11.1 \%$ of our patients but blindness was not detected in any of them. Conjunctival inflammation with granuloma formation and ulceration has been reported frequently [10] [25] [30]. Studies have reported frequency of $27 \%-100 \%$ of conjunctival inflammation in GPA patients [10] [25]. In our study $44.4 \%$, patients had conjunctivitis. Uveitis has been reported in $23.9 \%$ GPA patients in a study compared to $11.1 \%$ in our patients [7].

The orbit is frequently affected in ocular GPA presenting as proptosis, lid edema, diplopia and vision loss [1] [11] [29] [31]. In our study, none of the patients had proptosis. However, lid edema, decreased vison and lacrimal gland involvement were observed. Sino nasal involvement has been reported in up to $69 \%$ of GPA patients with orbital disease [10] [32]. We found sinonasal involvement in $77.8 \%$ of GPA patients with ocular disease. Several studies have shown association of sino-nasal manifestations with orbital disease and lacrimal gland involvement in GPA patients [10] [25] [32].

The predominant findings of orbital biopsy are reported to be chronic fibrotic changes rather than the classic triad of vasculitis, tissue necrosis and granulomatous inflammation [33]. In our patients, eye CT showed granuloma and orbital/lacrimal gland biopsy showed non-necrotizing granulomatous vasculitis. Orbital biopsy showed small vessel vasculitis in majority of the patients in some studies [34].

In our study patients responded to treatment with corticosteroids, IV CYC, methotrexate, rituximab and infliximab. Corticosteroid treatment along with IV CYC administration has been shown to increase the life expectancy of GPA patients which is otherwise a fatal disease [1] [5] [35]. Rituximab has shown to be more effective in relapsing patients and treatment of retro-orbital disease [36] [37] [38] [39]. Treatment with infliximab as an adjuvant therapy to CYC and methotrexate has been reported in the treatment of necrotizing and non-necrotizing scleritis [40] [41]. Necrotizing scleritis without appropriate treatment can lead to significant morbidity and mortality in GPA patients [42].

Comparison of ocular GPA patients with non-ocular GPA showed that ocular patients had significantly low risk of renal involvement compared to non-ocular GPA patients. There was concomitant ocular and sino-nasal involvement more 
frequently in ocular group compared to non-ocular GPA similar to previous studies, however in contrast to their finding; we did not find an association with of age at onset [25]. There was no significant association of ocular disease with other manifestations of GPA in our patients.

To the best of our knowledge this is the first study reporting the disease manifestations, therapy and outcome in ocular GPA patients from our region. Some of the limitations of our study are the small sample size and the cohort being drawn from a single institution which cannot be generalized to the entire Saudi population. The other limitation is the retrospective nature of our study.

\section{Conclusion}

The frequency of ocular manifestations in our GPA patients was similar to reports elsewhere, with the most frequent symptoms being eye pain and scleritis/episcleritis. The ocular involvement was associated with sino-nasal manifestations and significantly with low risk of renal involvement. Patients responded well to corticosteroid and IV CYC therapy and rituximab was more effective in relapsing patients. Ocular symptoms may be the first sign of GPA and early diagnosis and appropriate therapy is crucial to lowering the morbidity and vision loss in GPA patients.

\section{Conflicts of Interest}

The authors declare no conflicts of interest regarding the publication of this paper.

\section{References}

[1] Hoffman, G.S., Kerr, G.S., Leavitt, R.Y., et al. (1992) Wegener Granulomatosis: An Analysis of 158 Patients. Annals of Internal Medicine, 116, 488-498.

https://doi.org/10.7326/0003-4819-116-6-488

[2] Jennette, J.C. (2011) Nomenclature and Classification of Vasculitis: Lessons Learned from Granulomatosis with Polyangiitis (Wegener's Granulomatosis). Clinical and Experimental Immunology, 164, 7-10. https://doi.org/10.1111/j.1365-2249.2011.04357.x

[3] Ursea, R., De Castro, D., Bowen, T.J. and Chan, C.C. (2015) The Role of Conjunctival Biopsy in the Diagnosis of Granulomatosis with Polyangiitis. The Journal of Ophthalmic Inflammation and Infection, 5, Article No. 1.

https://doi.org/10.1186/s12348-014-0033-9

[4] Rothschild, P.R., Pagnoux, C., Seror, R., et al. (2013) Ophthalmologic Manifestations of Systemic Necrotizing Vasculitides at Diagnosis: A Retrospective Study of 1286 Patients and Review of the Literature. Seminars in Arthritis and Rheumatism, 42, 507-514. https://doi.org/10.1016/j.semarthrit.2012.08.003

[5] Kubaisi, B., Samra, A.K. and Foster, C.S. (2016) Granulomatosis with Polyangiitis (Wegener's Disease): An Updated Review of Ocular Disease Manifestations. Intractable \& Rare Diseases Research, 5, 61-69. https://doi.org/10.5582/irdr.2016.01014

[6] Puechal, X. (2007) Antineutrophil Cytoplasmic Antibody-Associated Vasculitides. Joint Bone Spine, 74, 427-435. https://doi.org/10.1016/j.jbspin.2007.02.001 
[7] Gheita, T.A. and Abd El Latif, E.M. (2019) Relationship of Ocular Presentation in Granulomatosis with Polyangiitis to Autoantibodies and Disease Activity. Zeitschrift für Rheumatologie, 78, 281-286. https://doi.org/10.1007/s00393-018-0495-5

[8] Generali, E., Cantarini, L. and Selmi, C. (2015) Ocular Involvement in Systemic Autoimmune Diseases. Clinical Reviews in Allergy \& Immunology, 49, 263-270. https://doi.org/10.1007/s12016-015-8518-3

[9] Rosenbaum, J.T. (2017) The Eye and Rheumatic Diseases. In: Firestein, G.S., Budd, R.C., Gabriel, S.E., McInnes, I.B. and O’Dell, J.R., Eds., Kelley and Firestein's Textbook of Rheumatology, Elsevier, Philadelphia, 645-653, Chapter 44.

[10] Jiang, B., Zhao, Y.Y. and Wei, S.H. (2013) Granulomatosis with Polyangiitis: The Relationship between Ocular and Nasal Disease. Ocular Immunology and Inflammation, 21, 115-118. https://doi.org/10.3109/09273948.2012.747618

[11] Holle, J.U., Voigt, C., Both, M., et al. (2013) Orbital Masses in Granulomatosis with Polyangiitis Are Associated with a Refractory Course and a High Burden of Local Damage. Rheumatology (Oxford), 52, 875-882.

https://doi.org/10.1093/rheumatology/kes382

[12] Tan, L.T., Davagnanam, I., Isa, H., et al. (2014) Clinical and Imaging Features Predictive of Orbital Granulomatosis with Polyangiitis and the Risk of Systemic Involvement. Ophthalmology, 121, 1304-1309. https://doi.org/10.1016/j.ophtha.2013.12.003

[13] Boukes, R.J. and de Vries-Knoppert, W.A. (1985) Lacrimal Gland Enlargement as One of the Ocular Manifestations of Wegener's Granulomatosis. Documenta Ophthalmologica, 59, 21-26. https://doi.org/10.1007/BF00162005

[14] Kiseleva, T.N., Grusha, I.O., Polunina, A.A., et al. (2009) Involvement of Lacrimal Organs in Wegener's Granulomatosis. Vestnik oftalmologii, 125, 33-36.

[15] Howe, L., D’Cruz, D., Chopdar, A., et al. (1995) Anterior Ischaemic Optic Neuropathy in Wegener's Granulomatosis. European Journal of Ophthalmology, 5, 277-279. https://doi.org/10.1177/112067219500500415

[16] Jennette, J.C., Falk, R.J., Bacon, P.A., et al. (2013) 2012 Revised International Chapel Hill Consensus Conference Nomenclature of Vasculitides. Arthritis \& Rheumatology, 65, 1-11. https://doi.org/10.1002/art.37715

[17] de Souza, F.H., Radu Halpern, A.S., Valente Barbas, C.S., et al. (2010) Wegener's Granulomatosis: Experience from a Brazilian Tertiary Center. Clinical Rheumatology, 29, 855-860. https://doi.org/10.1007/s10067-010-1408-4

[18] Reinhold-Keller, E., Beuge, N., Latza, U., et al. (2000) An Interdisciplinary Approach to the Care of Patients with Wegener's Granulomatosis: Long-Term Outcome in 155 Patients. Arthritis \& Rheumatology, 43, 1021-1032. https://doi.org/10.1002/1529-0131(200005)43:5<1021::AID-ANR10>3.0.CO;2-J

[19] Matteson, E.L., Gold, K.N. and Bloch, D.A. (1996) Long-Term Survival of Patients with Wegener's Granulomatosis from the American College of Rheumatology Wegener's Granulomatosis Classification Criteria Cohort. The American Journal of Medicine, 101, 129-134. https://doi.org/10.1016/S0002-9343(96)80066-0

[20] Pakrou, N., Selva, D. and Leibovitch, I. (2006) Wegener's Granulomatosis: Ophthalmic Manifestations and Management. Seminars in Arthritis and Rheumatism, 35, 284-292. https://doi.org/10.1016/j.semarthrit.2005.12.003

[21] Stone, J.H. and Wegener's Granulomatosis Etanercept Trial Research Group (2003) Limited versus Severe Wegener's Granulomatosis: Baseline Data on Patients in the Wegener's Granulomatosis Etanercept Trial. Arthritis \& Rheumatology, 48, 2299-2309. https://doi.org/10.1002/art.11075 
[22] Aldasoro-Cáceres, V., Aldasoro-Cáceres, I., Pérez-Moreiras, J.V., et al. (2014) C-ANCA Positive Necrotizing Scleritis and Multiple Sclerosis Compatible with Ocular Wegener: Treatment with Rituximab. Archivos de la Sociedad Española de Oftalmología, 89, 31-34. https://doi.org/10.1016/j.oftal.2012.06.020

[23] Solans-Laqué, R., Fraile, G., Rodriguez-Carballeira, M., et al. (2017) Clinical Characteristics and Outcome of Spanish Patients with ANCA Associated Vasculitides: Impact of the Vasculitis Type, ANCA Specificity, and Treatment on Mortality and Morbidity. Medicine (Baltimore), 96, e6083.

https://doi.org/10.1097/MD.0000000000006083

[24] Torres, R.M., Herreras, J.M., Becerra, E., et al. (2004) Ocular Presentation in Wegener Granulomatosis. Archivos de la Sociedad Española de Oftalmología, 79, 135-138. https://doi.org/10.4321/S0365-66912004000300008

[25] Hinojosa-Azaola, A., García-Castro, A., Juárez-Flores, A., et al. (2019) Clinical Significance of Ocular Manifestations in Granulomatosis with Polyangiitis: Association with Sinonasal Involvement and Damage. Rheumatology International, 39, 489-495. https://doi.org/10.1007/s00296-019-04242-7

[26] Akpek, E.K., Uy, H.S., Christen, W., et al. (1999) Severity of Episcleritis and Systemic Disease Association. Ophthalmology, 106, 729-731.

https://doi.org/10.1016/S0161-6420(99)90157-4

[27] Sainz de la Maza, M., Foster, C.S. and Jabbur, N.S. (1995) Scleritis Associated with Systemic Vasculitic Diseases. Ophthalmology, 102, 687-692. https://doi.org/10.1016/S0161-6420(95)30970-0

[28] Chung, S. and Monach, P.A. (2017) Anti-Neutrophil Cutoplasmic Antibody-Associated Vasculitis. In: Firestein, G.S., Budd, R.C., Gabriel, S.E., McInnes, I.B. and O'Dell, J.R., Eds., Kelley and Firestein's Textbook of Rheumatology, Elsevier, Philadelphia, 1541-1558, Chapter 89. https://doi.org/10.1016/B978-0-323-31696-5.00089-9

[29] Spalton, D.J., Graham, E.M., Page, N.G., et al. (1981) Ocular Changes in Limited Forms of Wegener's Granulomatosis. British Journal of Ophthalmology, 65, 553-563. https://doi.org/10.1136/bjo.65.8.553

[30] Fauci, A.S., Haynes, B.F., Katz, P., et al. (1983) Wegener's Granulomatosis: Prospective Clinical and Therapeutic Experience with 85 Patients for 21 Years. Annals of Internal Medicine, 98, 76-85. https://doi.org/10.7326/0003-4819-98-1-76

[31] Woo, T.L., Francis, I.C., Wilcsek, G.A., et al. (2001) Australasian Orbital and Adnexal Wagener's Study Group. Australasian Orbital and Adnexal Wegener's Granulomatosis. Ophthalmology, 108, 1535-1543.

https://doi.org/10.1016/S0161-6420(01)00655-8

[32] Tan, L.T., Davagnanam, I., Isa, H., et al. (2015) Clinical and Imaging Features of Lacrimal Gland Involvement in Granulomatosis with Polyangiitis. Ophthalmology, 122, 2125-2129. https://doi.org/10.1016/j.ophtha.2015.06.026

[33] Ahmed, M., Niffenegger, J.H., Jakobiec, F.A., et al. (2008) Diagnosis of Limited Ophthalmic Wegener's Granulomatosis: Distinctive Pathologic Features with ANCA Test Confirmation. International Ophthalmology, 28, 35-46. https://doi.org/10.1007/s10792-007-9109-y

[34] Kalina, P.H., Lie, J.T., Campbell, R.J., et al. (1992) Diagnostic Value and Limitations of Orbital Biopsy in Wegener's Granulomatosis. Ophthalmology, 99, 120-124. https://doi.org/10.1016/S0161-6420(92)32028-7

[35] Haubitz, M., Schellong, S., Göbel, U., et al. (1998) Intravenous Pulse Administration of Cyclophosphamide versus Daily Oral Treatment in Patients with Antineutrophil 
Cytoplasmic Antibody-Associated Vasculitis and Renal Involvement: A Prospective, Randomized Study. Arthritis \& Rheumatology, 41, 1835-1844.

https://doi.org/10.1002/1529-0131(199810)41:10<1835::AID-ART16>3.0.CO;2-Q

[36] Joshi, L., Tanna, A., McAdoo, S.P., et al. (2015) Long-Term Outcomes of Rituximab Therapy in Ocular Granulomatosis with Polyangiitis: Impact on Localized and Nonlocalized Disease. Ophthalmology, 122, 1262-1268.

https://doi.org/10.1016/j.ophtha.2015.01.016

[37] Bitik, B., Kılıç, L., Küçükşahin, O., et al. (2015) Retro-Orbital Granuloma Associated with Granulomatosis with Polyangiitis: A Series of Nine Cases. Rheumatology International, 35, 1083-1092. https://doi.org/10.1007/s00296-014-3179-8

[38] Joshi, L., Lightman, S.L., Salama, A.D., et al. (2011) Rituximab in Refractory Ophthalmic Wegener's Granulomatosis: PR3 Titers May Predict Relapse, But Repeat Treatment Can Be Effective. Ophthalmology, 118, 2498-2503.

https://doi.org/10.1016/j.ophtha.2011.06.009

[39] Baslund, B., Wiencke, A.K., Rasmussen, N., et al. (2012) Treatment of Orbital Inflammation with Rituximab in Wegener's Granulomatosis. Clinical and Experimental Rheumatology, 30, S7-S10.

[40] El-Shabrawi, Y. and Hermann, J. (2005) Anti-TNF Alpha Therapy in Chronic Necrotizing Scleritis Resistant to Standard Immunomodulatory Therapy in a Patient with Wegener's Granulomatosis. Eye, 19, 1017-1018.

https://doi.org/10.1038/sj.eye.6701712

[41] Kontkanen, M., Paimela, L. and Kaarniranta, K. (2010) Regression of Necrotizing Scleritis in Wegener's Granulomatosis after Infliximab Treatment. Acta Ophthalmologica, 88, e96-e97. https://doi.org/10.1111/j.1755-3768.2009.01546.x

[42] Wieringa, W.G., Wieringa, J.E., ten Dam-van Loon, N.H., et al. (2013) Visual Outcome, Treatment Results, and Prognostic Factors in Patients with Scleritis. Ophthalmology, 120, 379-386. https://doi.org/10.1016/j.ophtha.2012.08.005 